\title{
Security Constrained Optimal Power Flow Using Self-Organizing Hierarchical PSO with Time-Varying Acceleration Coefficients
}

\section{Jirawadee Polprasert ${ }^{1}$, Weerakorn Ongsakul ${ }^{1}$ and Dieu Vo $\mathrm{Ngoc}^{2 \star}$}

${ }^{1}$ Energy Field of Study, School of Environment, Resources and Development, Asian Institute of Technology, Klong Luang, Pathumthani 12120, Thailand ${ }^{2}$ Department of Power Systems Engineering, Ho Chi Minh City University of Technology, VNU-HCM, HCM City, Vietnam

\begin{abstract}
This paper proposes a self-organizing hierarchical particle swarm optimization (SOHPSO) with time-varying acceleration coefficients (TVAC) for solving security constrained optimal power flow (SCOPF) problem. The SCOPF is to minimize a total generation cost while satisfying generator operational constraints and network security constraints in which the minimum severity index is used to alleviate line loading. The proposed SOHPSO-TVAC re-initializes particle velocities whenever the population stagnates at local optima during the search and TVAC is used to enhance convergence to the global optimum solution. Test results on the IEEE 30 and 118 bus systems indicate that the proposed SCOPF renders a lower total generation cost in a faster convergence rate than the other heuristic methods, which is favorable for online implementation.
\end{abstract}

Keywords: Self-Organizing Hierarchical Particle Swarm Optimization (Soh-Pso); Time-Varying Acceleration Coefficients (TVAC); Security Constrained Optimal Power Flow (SCOPF); Severity Index (SI)

\section{List of Symbols and Abbreviations}

$a_{i}, b_{i}, c_{i}$

$S_{L}$

$S_{L, \max }$

$N B$

$N G$

$N L B$

NT

$N L$

$p$

$w$

$c_{1}, c_{2}$

rand $_{i}$

$P_{i}, Q_{i}$

$P_{G, i} Q_{G, i}$

$P_{D, i}, Q_{D, i}$

$V_{i}, V_{j}$

$\delta_{i}, \delta_{j}$

$\theta_{i j}$

$\mathrm{k}$

$k_{\max }$

$w_{\max }, w_{\min }$

$c_{1 i}, c_{1 f}$

$c_{2 i}, c_{2 f}$

$V_{d, \max }$

$\mathrm{R}$

$x_{d, \max }$
Cost function coefficients of unit $i, \$ / \mathrm{h}, \$ / \mathrm{MWh}$, and $\$ /$

$(\mathrm{MW})^{2} \mathrm{~h}$, respectively,

Line loading (MVA),

Line loading limit (MVA),

Number of buses,

Number of generating units,

Number of load buses,

Number of transformers,

Number of branches,

Population size,

Inertia weight factor,

Cognitive and social coefficients, respectively,

Uniformly distributed random numbers between 0 and 1 ,

Real and reactive power injection at the ith bus,

Total real and reactive power generation at the ith bus,

Total real and reactive power load at the ith bus,

Voltage magnitudes at bus $i$ and bus $j$,

Voltage angle of bus $i$ and bus $j$,

Angle of element i,j of bus admittance matrix,

Current iteration number,

Maximum number of iterations,

Maximum and minimum values of the inertia weight,

respectively,

Initial and final values of cognitive acceleration factors, respectively,

Initial and final values of social acceleration factors, respectively,

Maximum velocity limit in the dth dimension,

Chosen number of intervals in the dth dimension,

Maximum operating limits of the variable on each dimension,
Minimum operating limits of the variable on each dimension,
$K_{P}$

$K_{Q}$

$K_{s}$

$K_{V}$

$v_{i d}$

$x_{i d}$

pbest

gbest $_{g d}$

$x$

$u$

$F_{T}$

$F\left(P_{G, i}\right)$ Penalty weight of real power output of generator at slack bus

Penalty weight of reactive power output,

Penalty weight of line or transformer loading, Penalty weight of load bus voltage magnitude,

Velocity vectors of the ith particle of a d-dimensional search space,

Position vectors of the ith particle of a d-dimensional search space,

Best previous position of a particle of a d-dimensional search space,

Best among all particles in the group of $\mathrm{d}$-dimensional search space

Vector of dependent variables consisting of slack bus power, load bus voltages, reactive power outputs, and transmission line loadings,

Vector of independent variables consisting of generator voltages, real power outputs except at the slack bus, and transformer tap settings,

Total generator cost,

ith generating unit's generation cost which is a function of real power generation output.

*Corresponding author: Dieu Vo Ngoc, Department of Power Systems Engineering Ho Chi Minh City University of Technology, VNU-HCM, HCM City, Vietnam, Tel: +84-8 38645730; E-mail: vndieu@gmail.com

Received September 15, 2014; Accepted September 19, 2014; Published September 29, 2014

Citation: Polprasert J, Ongsakul W, Ngoc DV (2014) Security Constrained Optimal Power Flow Using Self-Organizing Hierarchical PSO with TimeVarying Acceleration Coefficients. Global J Technol Optim 5: 163. doi: $10.4172 / 2229-8711.1000163$

Copyright: () 2014 Polprasert J, et al. This is an open-access article distributed under the terms of the Creative Commons Attribution License, which permits unrestricted use, distribution, and reproduction in any medium, provided the original author and source are credited. 


\section{Introduction}

Optimal power flow (OPF) has been widely used for economic and secure power system operation. OPF function is to determine the optimal settings for control variables such as active power outputs and voltages, shunt capacitors/reactors, and transformer tap-settings which minimize total generator fuel cost while satisfying generator operating and network constraints.

OPF is generally a large-dimension nonlinear and highlyconstrained optimization problem. Several techniques have been proposed to solve the OPF problem including Newton-based techniques, nonlinear programming, quadratic programming, linear programming and interior point methods [1]. These methods rely on convex and continuous fuel-cost function to obtain the optimum solution. Therefore, they may face some difficulty when non-convex characteristics of generator fuel-cost function are considered [2]. To overcome these barriers, many heuristic algorithms such as genetic algorithm (GA), tabu search (TS), hybrid tabu search and simulated annealing (TS/SA) and evolutionary programming (EP), which are used to solve security-constrained optimal power flow (SCOPF) problems, have been proposed to solve the OPF problem. The improved PSO-based contingency constrained ELD (CCELD) solution gives the optimal setting of all controllable variables for a static power setting loading condition [3]. In addition, GA-based algorithm has been proposed for OPF problem for security enhancement and line overload is removed by means of generation re-dispatching and adjustment of phase shifter transformer [4]. For EP-based security constrained economic load dispatch (ELD), the contingency constraints are not taken into account [5].

In this paper, the SOHPSO with TVAC is proposed to solve the SCOPF problem. SOHPSO with TVAC is designed to avoid premature convergence during the early stages of the search and is capable of determining the global optimum solution for a range of constraints and objective functions [6]. The particle velocities are reinitialized whenever the population stagnates at a local minimum during the search. The proposed method is tested on IEEE 30-bus and 118bus systems and compared to those of evolutionary programming (CEP) with Gaussian mutation with scaled cost [2], basic particle swarm optimization (BPSO), PSO with Time-Varying Inertia Weight factor method (PSO-TVIW), PSO with Time-Varying Acceleration Coefficients (PSO-TVAC), and Self-Organizing Hierarchical PSO with TVAC (SOHPSO-TVAC).

\section{Problem Formulation}

\section{Objective function}

The objective function of the SCOPF problem is to

Minimize $F_{T}=\sum_{i=1}^{N G} f_{i}\left(P_{G, i}\right)$

\section{Constraints}

(1) Power balance equations

$$
\begin{gathered}
P_{i}=P_{G, i}-P_{D, i}=\sum_{j=1}^{N B}\left|V_{i} V_{j} Y_{i j}\right| \cos \left(\theta_{i j}-\delta_{i}+\delta_{j}\right) \\
Q_{i}=Q_{G, i}-Q_{D, i}=-\sum_{j=1}^{N B}\left|V_{i} V_{j} Y_{i j}\right| \sin \left(\theta_{i j}-\delta_{i}+\delta_{j}\right)
\end{gathered}
$$

(2) Generation operating constraints

$\left|V_{G, i}^{\min }\right| \leq\left|V_{G, i}\right| \leq\left|V_{G, i}^{\max }\right|, i \in N G$

$$
\begin{gathered}
P_{G, i}^{\min } \leq P_{G, i} \leq P_{G, i}^{\max }, i \in N G \\
Q_{G, i}^{\min } \leq Q_{G, i} \leq Q_{G, i}^{\max }, i \in N G
\end{gathered}
$$

(3) Network constraints

$$
\begin{aligned}
& t_{i}^{\min } \leq t_{i} \leq t_{i}^{\max }, i \in N G \\
& \left|V_{L, i}^{\min }\right| \leq\left|V_{L, i}\right| \leq\left|V_{L, i}^{\max }\right|, \quad i \in N L B \\
& \left|S_{L, i}\right| \leq S_{L, i}^{\max }, \quad i \in N L \text { (n-1 contingency) }
\end{aligned}
$$

The contingency ranking is a measure to evaluate the relative severity of a contingency to line overload. The severity index (SI) used for ranking contingency is [1]:

$$
S I=\sum_{i=1}^{N L}\left(\frac{S_{L, i}}{S_{L, i}^{\max }}\right)^{2}
$$

\section{PSO Algorithms for SCOPF Problem}

\section{Basic PSO}

Particle swarm optimization (PSO) provides a population-based search procedure in which individuals called particles change their position (state) with time. In a PSO system, particles fly around in a multidimensional search space. During the flight, each particle adjusts its position according to its own experience (personal best: pbest), and according to the experience of a neighboring particle (global best: gbest), leading to the best position encountered by itself and its neighbor. The modified velocity and position of each particle are calculated:

$$
\begin{aligned}
& \begin{aligned}
v_{i d}^{k+1}=w \times v_{i d}^{k}+c_{1} \times \text { rand }_{1} \times\left(\text { pbest }_{i d}-\right. & \left.x_{i d}\right) \\
& +c_{2} \times \text { rand }_{2} \times\left(\text { gbest }_{g d}-x_{i d}\right)
\end{aligned} \\
& x_{i d}^{k+1}=x_{i d}^{k}+v_{i d}^{k+1}
\end{aligned}
$$

\section{PSO with Time-Varying Inertia Weight (TVIW)}

The modified velocity of each particle is calculated:

$$
v_{i d}^{k+1}=C *\left[w \times v_{i d}^{k}+c_{1} \times \text { rand }_{1} \times\left(\text { pbest }_{i d}-x_{i d}\right)+c_{2} \times \text { rand }_{2} \times\left(\text { gbest }_{g d}-x_{i d}\right)\right]
$$

The concept of time varying inertial weight (TVIW) was introduced in [6]. The use of linearly decreasing inertia weight factor $(w)$ is to improve performance in searching for optimal solution. Its value is decreased linearly from wmax to wmin as iteration grows. Suitable selection of the inertia weight provides a balance between global and local exploration abilities, and results in less iteration to find an optimal solution. w decreases linearly from 0.9 to 0.4 during iteration. Its value is set as:

$$
w=\left(w_{\max }-w_{\min }\right) \times\left(\frac{k_{\max }-k}{k_{\max }}\right)+w_{\min }
$$

To improve the convergence rate, the constriction factor $(C)$ is analyzed by the Eigen value analysis expressed as:

$$
C=\frac{2}{\left|2-\phi-\sqrt{\phi^{2}-4 \phi}\right|} \text {, where } 4.1 \leq \Phi \leq 4.2
$$

The convergence characteristics of the system are controlled by $\Phi$. As $\Phi$ increases, the factor $C$ decreases leading to slower convergence rate because population diversity is reduced.

\section{PSO with Time-Varying Acceleration Coefficients (TVAC)}

Even though the TVIW-PSO can determine a better solution in a fast convergence rate, its ability to fine tune the optimal solution 
is lacking because of diversity at the end of the search [6]. TVAC enhances the global search in the early stage and persuades the particles to converge toward the global optimum at the end of the search. By changing the acceleration coefficients $\mathrm{cl}$ and $\mathrm{c} 2$ with TVAC, the cognitive component is reduced while the social component increases as the search proceeds. A large cognitive component and a small social component allow the particles to move around the search space instead of moving toward the population best during early stages [7]. By contrast, the particles are allowed to converge to the global optimum in the latter part of optimization. Mathematically, the acceleration coefficients can be formulated as follow:

$$
\begin{aligned}
& c_{1}=\left(c_{1 f}-c_{1 i}\right)\left(\frac{k}{k_{\max }}\right)+c_{1 i} \\
& c_{2}=\left(c_{2 f}-c_{2 i}\right)\left(\frac{k}{k_{\max }}\right)+c_{2 i}
\end{aligned}
$$

\section{Proposed SOHPSO with TVAC}

The proposed SOHPSO-TVAC is a novel concept of PSO that effectively finds the global optima. Here the previous velocity term is made to zero and particles may rush to a local optimal solution. With this modification, particles stagnate due to lack of momentum to find the global optimal solution. To overcome the limitation, the velocity vector of a particle is reinitialized with a random velocity whenever it stagnates during the search. This modification is represented by the concept of reinitialized velocity. The updating velocity each particle can be mathematically represented using the current velocity and the distance from pbestid and gbestgd as follows [8]:

$$
v_{i d}^{k+1}=c_{1} \times \text { rand }_{1} \times\left(\text { pbest }_{i d}-x_{i d}\right)+c_{2} \times \text { rand }_{2} \times\left(\text { gbest }_{g d}-x_{i d}\right)
$$

The pseudocode for the SOHPSO-TVAC method is as follows:

Initialize the population

Velocity updating using Eq. (11)

$$
\begin{aligned}
& \text { If } v_{i d}=0 \\
& \text { if } r \text { and }<0.5 \\
& v_{i d}=-r \text { and }^{*} v_{d, \max } \\
& \text { else } v_{i d}=-r \text { and }{ }^{\star} v_{d, \text { max }} \\
& \text { end ifend if } \\
& v_{i d}=\operatorname{sign}\left(v_{i d}\right)^{*} \min \left(a b s\left(v_{i d,}, v_{d, \max }\right)\right)
\end{aligned}
$$

Position updating using Eq. (12)

in which, $\operatorname{sign}\left(v_{i d}\right)=1$ if $v_{i d} \geq 0$ and $\operatorname{sign}\left(v_{i d}\right)=-1$ otherwise. The maximum velocity limit for the $d$ th dimension is computed as following:

$$
V_{d, \text { max }}=\frac{\left(x_{d, \text { max }}-x_{d, \text { min }}\right)}{R}
$$

\section{Selection of parameter}

Based on empirical studies on a number of mathematical benchmarks [6], an improved optimum solution has reported the best range of variation as 2.5-0.5 for $c_{1}$ and 0.5-2.5 for $c_{2}$. Note that a maximum initial value of the cognitive coefficient $c$ is intended to diversify the search whereas an initial minimum social coefficient $c_{2}$ is to avoid the premature convergence. Local search space is reduced as $c_{1}$

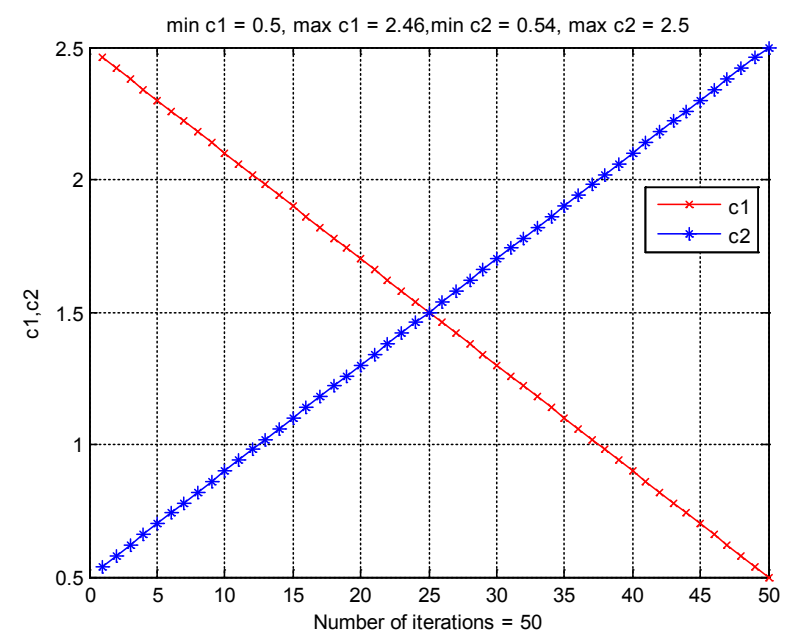

Figure 1: Characteristic of cognitive and social coefficients for TVAC.

decreases and $c_{2}$ increases to accelerate the solution towards the global convergence as shown in Figure 1.

\section{Procedure of the proposed SOHPSO with TVAC algorithm for SCOPF problem}

The procedure of the proposed SOHPSO algorithm can be described in the following steps:

Step 1: Compute load flow analysis with each single line outage and calculate the corresponding severity index by using Eq. (10).

Step 2: Select the two highest severity index cases for running SCOPF by SOHPSO with TVAC.

Step 3: Initialize randomly the particles according to the limit of each unit (except slack bus) including individual dimension, searching points and velocities. The new velocity strategy equation has been formulated and the maximum and minimum velocity limits of each variable are calculated using Eq. (20) and Eq. (21) as follows:

$$
V_{d, \text { min }}=-\frac{\left(x_{d, \text { max }} x_{d, \text { min }}\right)}{\left(x_{d, \text { max }}-x_{d, \text { min }}\right)}
$$

Step 4: Set an iteration counter $k=0$ and generate random particles, then generated random initial velocities of all particles.

Step 5: Evaluate each particle using fitness functions and determines pbest and gbest.

Step 6: Update the iteration counter $k=k+1$.

Step 7: Update velocity of each particle and re-initialization of velocity and check the limits on velocity. To control excessive roaming of particles, velocity is made to lie between $-V_{d, \max }$ and $V_{d, \max }$

Step 8: Update position of each particle by using Eq. (12) and check the operating limits of generator.

Step 9: Update pbest and gbest. If the evaluation value of each particle is better than the previous pbest, the current value is set to be pbest. If the best pbest is better than gbest, the value is set to be gbest.

Step 10: The objective function from Eq. (1) is extended by the 
penalty function of real power generation of generator at slack bus, line and transformer loading, and load bus voltage magnitude and total operating cost is augmented by non-negative penalty terms $\left(K_{P}, K_{Q}, K_{S}\right.$, $K_{V}$, and $K_{T}$ ). Thus, augmented fitness function is:

$$
\begin{aligned}
F_{T}^{*}=F_{T}+K_{P} & \times\left(P_{G 1}-P_{G 1}^{\lim }\right)^{2}+K_{Q} \times \sum_{i=1}^{N G}\left(Q_{G, i}-Q_{G, i}^{\lim }\right)^{2} \\
& +K_{S} \times \sum_{i=1}^{N L}\left(S_{L, i}-S_{L, i}^{\max }\right)^{2}+K_{V} \times \sum_{i=1}^{N L B}\left(V_{L, i}-V_{L, i}^{\lim }\right)^{2}
\end{aligned}
$$

Step 11: Maximum number of iterations is taken as the stopping criterion. If one of the stopping criteria is satisfied, then stop; else go to step 4 .

Step 12: gbest of the best solution is the best particle which obtains the optimum objective function value and satisfying all the constraints (Figure 2).

\section{Numerical Results}

The proposed SOHPSO-TVAC for SCOPF problem is tested on the IEEE 30-bus system [7] and IEEE 118-bus system [8]. The IEEE 30bus system has six generators and 41 transmission lines and the IEEE 118-bus system has 54 generator buses and 186 transmission lines. The contingency analysis is made under the base load conditions to identify the severe contingencies. In this paper, the first two severe contingencies are considered for security assessment. If the algorithm relieves overload on two-top severe contingencies, the algorithm can relieve overload on lines for less severe contingencies. Three different cases are considered for the study. In the first case, the proposed SOHPSO-TVAC is applied to obtain the optimal-control variables on IEEE 30-bus system without

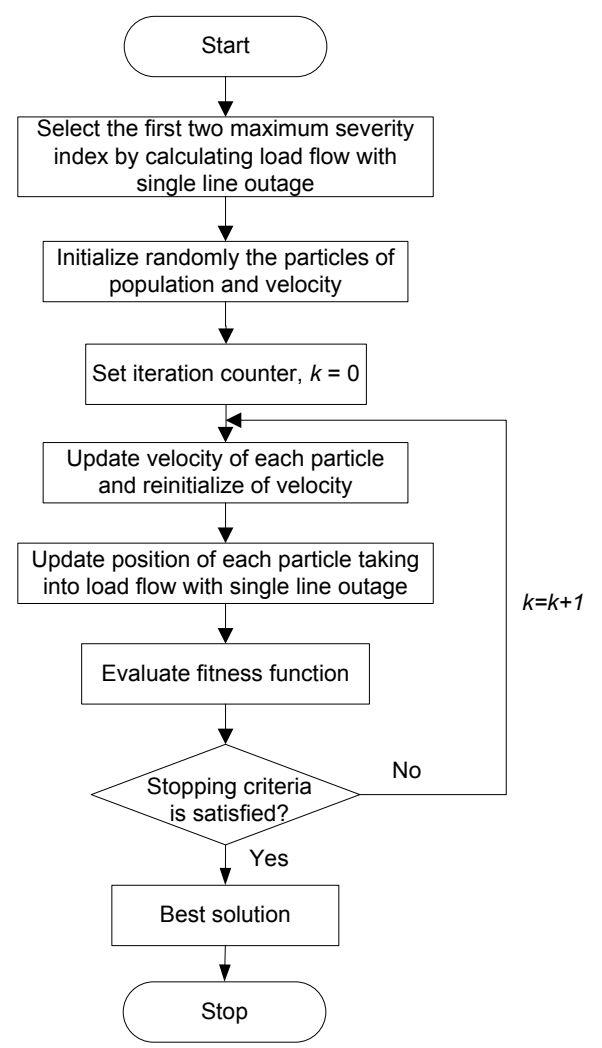

Figure 2: The flowchart of the SOHPSO with TVAC for SCOPF problem. line-outage. The upper and lower voltage limits at all buses except the slack bus are set to 1.05 and 0.95 p.u., respectively. The slack bus voltage is fixed to its specified value of 1.06 p.u. In addition, the line flows are computed using N-R load flow method. In the second case, the proposed algorithm is applied to alleviate overloads under line outage through generator rescheduling on IEEE 30-bus system. Simulation parameters of PSO algorithms are shown in Table 1. For the third case, the proposed method is tested on IEEE 118-bus system and applied to alleviate overloads under line outage through generator scheduling. The algorithm of the proposed method is implemented in MATLAB 7.6 and run on a $1.87 \mathrm{GHz}$ PC with $3 \mathrm{~GB}$ of RAM (Table 1).

\section{Base case of OPF for IEEE 30-bus (without line outage)}

In Table 2, the total generator fuel cost obtained from the proposed method is lower than genetic algorithm (GA) [4], parallel self-adaptive differential evolution with augmented Lagrange multiplier (pSADE_ ALM) [9], classical economic dispatch and standard load flow (EDLF) [10], Dommel-Tinney (DT) [7], modified particle swarm optimization (MPSO) [11], PSO [12], improved particle swarm optimization (IPSO) [13], and Tabu Search (TS) [14] (Table 2). In addition, the proposed SOHPSO-TVAC renders better solutions and requires less CPU time than basic particle swarm optimization (BPSO), particle swarm optimization with time-varying inertia weight (PSO-TVIW), and particle swarm optimization with time-varying acceleration coefficients (PSO-TVAC) as shown in Table 3. In Figure 3, SOHPSOTVAC converges to a better solution in a faster manner than BPSO, PSO-TVIW, and PSO-TVAC.

\section{SCOPF on IEEE 30-bus system}

From the contingency analysis on IEEE 30-bus system, it is found that each line outage from line 1-3 and line 3-4 result in overload in other lines. The power on the overloaded lines and the calculated value of severity index for each contingency are given in Table 4 . Two-line outages 1-3 and 3-4 are the most severity. Table 5 shows all results obtained in 50 trial runs and giving zero-severity indices but the minimum fuel cost and CPU time are obtained by the proposed SOHPSO-TVAC based SCOPF. The results have shown that the SOHPSO-TVAC algorithm achieves the lower total generator fuel cost with zero-severity indices when compared with other methods.

\section{SCOPF on IEEE 118-bus system}

In this case, the proposed algorithm is used for corrective control under a contingency state. The proposed method is applied to alleviate the lines overload under contingency condition on the IEEE 118-bus system. From the contingency analysis, it is found that line outages 8-5 and 64-65 result in overload on other lines. The power flow on the overloaded line and the calculated value of severity index for each contingency are given in Table 6. The IEEE 118-bus test system consists of 54 generator buses and 186 transmission lines [8,15-17]. Contingency analysis is performed on the system and the top two-severe contingency

\begin{tabular}{|l|c|c|c|c|c|}
\hline \multicolumn{1}{|c|}{ Approach } & $\begin{array}{c}\text { No. of } \\
\text { particles }\end{array}$ & $\boldsymbol{k}_{\max }$ & $\boldsymbol{w}$ & $\boldsymbol{c}_{\mathbf{1}}$ & $\boldsymbol{c}_{\mathbf{2}}$ \\
\hline BPSO & 50 & 50 & 0.5 & 2 & 2 \\
\hline PSO-TVIW & 50 & 50 & $\begin{array}{c}0.9-0.4 \\
\text { (decreasing) }\end{array}$ & 2 & 2 \\
\hline PSO-TVAC & 50 & $\begin{array}{c}0.9-0.4 \\
\text { (decreasing) }\end{array}$ & $\begin{array}{c}2.5-0.5 \\
\text { (decreasing) }\end{array}$ & $\begin{array}{c}0.5-2.5 \\
\text { (increasing) }\end{array}$ \\
\hline SOHPSO-TVAC & 50 & 50 & 0 & $\begin{array}{c}2.5-0.5 \\
\text { (decreasing) }\end{array}$ & $\begin{array}{c}0.5-2.5 \\
\text { (increasing) }\end{array}$ \\
\hline
\end{tabular}

Table 1: Simulation parameters of PSO algorithms. 
Citation: Polprasert J, Ongsakul W, Ngoc DV (2014) Security Constrained Optimal Power Flow Using Self-Organizing Hierarchical PSO with TimeVarying Acceleration Coefficients. Global J Technol Optim 5: 163. doi: 10.4172/2229-8711.1000163

Page 5 of 6

\begin{tabular}{|c|c|c|c|c|c|c|c|c|c|}
\hline Power Output (MW) & GA [4] & pSADE_ALM [9] & TS [14] & EDLF [10] & DT [7] & MPSO [11] & PSO [12] & IPSO [13] & SOHPSO TVAC \\
\hline$P_{G 1}$ & 179.39 & 123.61 & 176.04 & 192.65 & 138.56 & 176.44 & - & 198.69 & 177.72 \\
\hline$P_{G 2}$ & 48.83 & 61.92 & 48.76 & 48.92 & 57.56 & 48.82 & - & 36.16 & 47.78 \\
\hline$P_{G 5}$ & 21.84 & 30.40 & 21.56 & 19.26 & 24.56 & 21.48 & - & 17.64 & 25.37 \\
\hline$P_{G 6}$ & 21.75 & 34.74 & 22.05 & 10.58 & 35.00 & 21.99 & - & 11.45 & 14.74 \\
\hline$P_{G 11}$ & 12.05 & 20.66 & 12.44 & 10.79 & 17.93 & 12.17 & - & 12.15 & 13.2 \\
\hline$P_{G 13}$ & 12.36 & 18.30 & 12.00 & 12.24 & 16.91 & 12.00 & - & 11.87 & 14.66 \\
\hline$P_{\text {LosS }}$ & - & - & - & 11.04 & 7.13 & - & 10.08 & 9.12 & 9.49 \\
\hline Total cost $(\$ / \mathrm{h})$ & 803.5 & 802.40 & 802.29 & 805.45 & 813.74 & 802.40 & 802.19 & 827.13 & 802.03 \\
\hline CPU Time (sec) & - & - & - & - & - & - & - & 926 & 29.43 \\
\hline
\end{tabular}

Table 2: Comparison of total cost and computational time of IEEE 30-bus system (without line-outage).

\begin{tabular}{|l|c|c|c|}
\hline \multicolumn{1}{|c|}{ Power Output (MW) } & BPSO & PSO-TVIW & PSO-TVAC \\
\hline$P_{G 1}$ & 176.911 & 175.962 & 172.44 \\
\hline$P_{G 2}$ & 50.384 & 45.786 & 49.105 \\
\hline$P_{G 5}$ & 21.424 & 20.636 & 177.716 \\
\hline$P_{G 6}$ & 20.138 & 27.757 & 25.767 \\
\hline$P_{G 11}$ & 12.07 & 10.354 & 14.742 \\
\hline$P_{G 13}$ & 12.568 & 13.683 & 13.2 \\
\hline$P_{\text {LosS }}$ & 11.925 & 10.738 & 17.147 \\
\hline Total cost (\$/h) & 803.13 & 802.11 & 15.505 \\
\hline CPU Time (sec) & 35.15 & 33.756 & 9.620 \\
\hline
\end{tabular}

Table 3: Comparison of different PSO algorithms of IEEE 30-bus system (without line-outage).

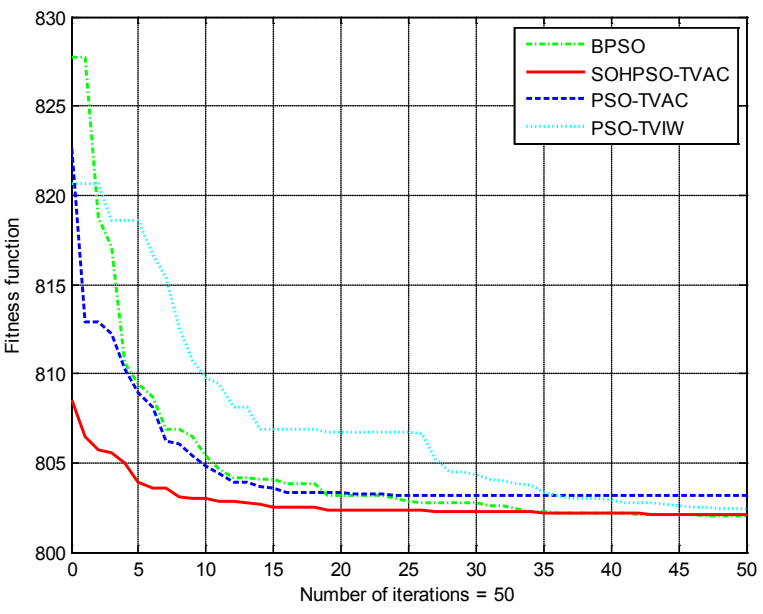

Figure 3: Convergence characteristics of different PSO.

\begin{tabular}{|c|c|c|c|c|}
\hline Line outage & $\begin{array}{c}\text { Overloaded } \\
\text { lines }\end{array}$ & $\begin{array}{c}\text { Line flow } \\
\text { (MVA) }\end{array}$ & $\begin{array}{c}\text { Line flow limit } \\
\text { (MVA) }\end{array}$ & Severity Index \\
\hline \multirow{3}{*}{$1-3$} & $1-2$ & 270.81 & 125 & \multirow{2}{*}{7.562} \\
\cline { 2 - 4 } & $2-4$ & 86.44 & 75 & \multirow{2}{*}{7.413} \\
\cline { 2 - 4 } & $2-6$ & 93.08 & 75 & \\
\hline \multirow{3}{*}{$3-4$} & $1-2$ & 267.89 & 125 & \\
\cline { 2 - 4 } & $2-4$ & 85.39 & 75 & \\
\cline { 2 - 4 } & $2-6$ & 95.57 & 75 & \\
\hline
\end{tabular}

Table 4: Summary of contingency analysis for IEEE 30-bus System.

cases are shown in Table 6 along with the overloaded lines and the severity index. The CEP, FEP, MFEP, CPSO, PSO-TVIW, PSO-TVAC and SOHPSO-TVAC based SCOPF are applied to reschedule the generator output to minimize generator fuel cost with the least severity index. Table 7 shows from 50 independent trail runs and the proposed

\begin{tabular}{|c|c|c|c|c|c|}
\hline Approach & Line outage & $\begin{array}{l}\text { Best Cost } \\
(\$ / h)\end{array}$ & $\begin{array}{l}\text { Average } \\
\text { Cost (\$/h) }\end{array}$ & $\begin{array}{l}\text { Worst Cost } \\
(\$ / h)\end{array}$ & $\begin{array}{l}\text { CPU Time } \\
\text { (sec) }\end{array}$ \\
\hline MFEP [2] & \multirow{7}{*}{$1-3$} & 1245.1 & - & - & 59.58 \\
\hline CEP [2] & & 1241.1 & - & - & 59.06 \\
\hline FEP [2] & & 1233.4 & - & - & 57.38 \\
\hline BPSO & & 1217.6 & 1263.9 & 1297.3 & 57.68 \\
\hline PSO-TVIW & & 1237.5 & 1288.3 & 1321.5 & 55.32 \\
\hline PSO-TVAC & & 1224.3 & 1295.6 & 1345.7 & 49.55 \\
\hline $\begin{array}{l}\text { SOHPSO- } \\
\text { TVAC }\end{array}$ & & 1215.4 & 1321.5 & 1387.1 & 45.95 \\
\hline FEP [2] & \multirow{7}{*}{$3-4$} & 1242.0 & - & - & 61.25 \\
\hline MFEP [2] & & 1240.6 & - & - & 63.37 \\
\hline CEP [2] & & 1229.7 & - & - & 62.15 \\
\hline BPSO & & 1246.9 & 1254.3 & 1267.5 & 62.82 \\
\hline PSO-TVIW & & 1237.8 & 1245.5 & 1252.3 & 61.36 \\
\hline PSO-TVAC & & 1232.4 & 1241.8 & 1248.1 & 60.57 \\
\hline $\begin{array}{l}\text { SOHPSO- } \\
\text { TVAC }\end{array}$ & & 1228.6 & 1337.4 & 1406.8 & 60.43 \\
\hline
\end{tabular}

Table 5: Comparison of best results IEEE 30-bus system (with line outage either no. 1-3 or 3-4).

\begin{tabular}{|c|c|c|c|c|}
\hline Line outage & $\begin{array}{c}\text { Overloaded } \\
\text { lines }\end{array}$ & $\begin{array}{c}\text { Line flow } \\
\text { (MVA) }\end{array}$ & $\begin{array}{c}\text { Line flow limit } \\
\text { (MVA) }\end{array}$ & Severity index \\
\hline \multirow{3}{*}{$8-5$} & $12-14$ & 107.187 & 100 & \\
\cline { 2 - 4 } & $13-15$ & 103.659 & 100 & \multirow{2}{*}{6.2181} \\
\cline { 2 - 4 } & $12-16$ & 143.441 & 130 & \multirow{2}{*}{4.1663} \\
\cline { 2 - 4 } & $15-17$ & 223.657 & 200 & \multirow{2}{*}{4.130} \\
\cline { 2 - 4 } & $16-17$ & 160.625 & 100 & \\
\hline \multirow{3}{*}{$64-65$} & $65-66$ & 172.655 & 100 & \\
\hline
\end{tabular}

Table 6: Summary of contingency analysis for IEEE 118-bus System.

SOHPSO with TVAC method obtains the best total fuel cost than the other methods.

\section{Conclusion}


Citation: Polprasert J, Ongsakul W, Ngoc DV (2014) Security Constrained Optimal Power Flow Using Self-Organizing Hierarchical PSO with TimeVarying Acceleration Coefficients. Global J Technol Optim 5: 163. doi: 10.4172/2229-8711.1000163

Page 6 of 6

\begin{tabular}{|c|c|c|c|c|c|}
\hline Method & Line Outage & Best Cost (\$/h) & Average Cost $(\$ / h)$ & Worst Cost (\$/h) & CPU Time (sec) \\
\hline MFEP [2] & \multirow{7}{*}{$8-5$} & 23,882 & - & - & 110.43 \\
\hline FEP [2] & & 23,528 & - & - & 108.57 \\
\hline CEP [2] & & 22,213 & - & - & 109.35 \\
\hline BPSO & & $22,569.4$ & $24,789.3$ & $27,342.9$ & 109.75 \\
\hline PSO-TVIW & & $22,441.3$ & $23,478.8$ & $24,552.4$ & 107.84 \\
\hline PSO-TVAC & & $23,347.4$ & $24,463.5$ & $25,532.6$ & 108.21 \\
\hline SOHPSO-TVAC & & $22,117.3$ & $23,462.9$ & $24,348.5$ & 107.29 \\
\hline MFEP [2] & \multirow{7}{*}{$64-65$} & 24,045 & - & - & 112.56 \\
\hline FEP [2] & & 24,042 & - & - & 110.46 \\
\hline CEP [2] & & 22,712 & - & - & 111.32 \\
\hline BPSO & & 21,996 & $22,754.3$ & $23,452.8$ & 110.95 \\
\hline PSO-TVIW & & $22,188.14$ & $23,475.3$ & $24,772.5$ & 109.71 \\
\hline PSO-TVAC & & $22,934.69$ & $24,389.2$ & $25,843.6$ & 110.63 \\
\hline SOHPSO-TVAC & & $21,832.56$ & $23,248.4$ & $24,432.5$ & 109.48 \\
\hline
\end{tabular}

Table 7: Comparison of best results on IEEE 118-bus system (with line outage either no. 8-5 or 64-65).

In this paper, a self-organizing hierarchical particle swarm optimization with time-varying acceleration coefficients has been efficiently solving SCOPF problem. The proposed SOHPSO-TVAC method reinitializes the velocity vector when it stagnates and properly balances between the local and global exploration. Test results on the IEEE 30-bus and 118-bus systems indicate that the proposed SCOPF method can find better solutions than the other methods in a faster convergence. Therefore, the proposed SOHPSO with TVAC is potentially suitable for online SCOPF implementation.

\section{Acknowledgement}

This research is funded by Vietnam National University HoChiMinh City (VNUHCM) under grant number C2014-20-24.

\section{References}

1. Abido MA (2002) Optimal Power Flow Using Particle Swarm Optimization Electric Power Components and Systems 24: 563-571.

2. Baskar G, Mohan MR (2009) Contingency constrained economic load dispatch using improved particle swarm optimization for security enhancement. Electric Power System Research 79: 615-621.

3. Pancholi RK., Swarup KS (2002) Particle Swarm Optimization for security constrained economic dispatch. Proceedings of Intelligence Sensing and Information (ICISIP) 7-12.

4. Devaraj D, Yegnanarayana B (2005) Genetic algorithm - based optimal power flow for security enhancement. IEE. Proceeding-Generation Transmission Distribution 152: 899-905.

5. Somasundaram P, Kuppusamy K., Devi RPK. (2004) Evolutionary programming based security constrained optimal power flow. Elect. Power Syst. Research 72: $137-145$
6. Ratnaweera A, Halgamuge SK (2004) Self-Organizing Hierarchical Particle Swarm Optimizer with Time-Varying Acceleration coefficients. IEEE Trans. on Evolutionary Computation 8: 240-255.

7. Alsac O, Stott B (1974) Optimal load flow with steady - state security. IEEE Trans. Power Apparatus Syst. 93: 745-751.

8. IEEE-118 bus system (1996), United states Available at: http://www ee.washington.edu.

9. Thitithamrongchai C, Eua-arporn B (2008) Security-constrained Optimal Power Flow: A parallel Self-adaptive Differential Evolution Approach. Elec. Power Comp Syst 36: 280-298.

10. Osman MS, Abo-Sinna MA., Mousa AA (2004) A solution to the optimal power flow using genetic algorithm. Applied Mathematics and Computation 155: 391-405.

11. Hajian-Hoseinabadi H, Hosseini SH, Hajian M (2008) Optimal Power Flow Solution by a Modified Particle Swarm Optimization Algorithm. Proceedings of Universities Power Engineering Conference (UPEC): 1-4

12. Kumari MS, Priyanka G, Sydulu M (2007) Comparison of Genetic Algorithms and Particle Swarm Optimization for Optimal Power Flow Including FACTS devices. Proceedings of Power Tech, IEEE Lausanne: 1105-1110.

13. Weibing Liu, Min Li, Xianjia W (2009) An Improved Particle Swarm Optimization Algorithm for Optimal Power Flow. Proceedings of Power Electronics and Motion Control Conference: 2448-2450.

14. Shi Y (2004) Particle Swarm Optimization. IEEE Neural Network Society: 8-13.

15. Roa-Sepulveda CA. Pavez-Lazo BJ (2003) A solution to the optimal power flow using simulated annealing. Elec. Power and Energy Syst 25: 47-57.

16. Medicherla TKP, Billington R, Sachdev MS (1979) Generation rescheduling and load shedding to alleviate overload-analysis. IEEE Trans. Power Syst 98 1879-1884.

17. Udupa AN, Purushothama GK, Parthasarathy K., Thukaram D (2001) A fuzzy control for network overload alleviation. Elect. Power Energy Syst 23: 119-129. 